\title{
Molecular Clustering and Velocity Increase in Converging-Diverging Nozzle in MD Simulation
}

\author{
Jeoungsu $\mathrm{Na}^{1}$, Jaehawn Lee ${ }^{2}$, Changil Hong ${ }^{2}$, Suhee Kim² \\ ${ }^{1} R \& D$ Department of NaJen, Co. LTD, Korea \\ ${ }^{2}$ Dept. of Computer Engineering, Hoseo University, Asan Korea
}

\begin{abstract}
A Molecular dynamics simulation in a converging-diverging nozzle was performed to study molecular collisions and their influence to average flow velocity according to a variety of vacuum levels. The static pressures and the dynamic pressure exerted by the molecule collision on the selected walls were compared to figure out the intensity variances of the directional flows. With pressure differences constant between the entrance and the exit of the nozzle, The numerical experiment was performed for molecular velocities and directional flows. The result shows that the velocities are increased at the nozzle exit as the vacuum level gets higher in that area because of less molecular collisions
\end{abstract}

\section{Introduction}

Hydroelectric generator converts potential energy of high positioned water into electricity using gravitational force with over $90 \%$ of total efficiency. It is high value compared to other electric power generation such as $38 \%$ efficiency of thermal power plants. However, for stable operation of the power plants and to make sufficient electricity, thermal or nuclear power plants are used widely even though the low efficiency. They use high pressured steam jet to rotating turbine by the Rankine cycle, which has condensing stage for recirculation of liquid water. In the condensing state, however, we have been ignored the discharging heat energy without doubt. Especially, for these power plants, the energy of discharging cooling water is over $50 \%$, that is the energy lease to sea or atmosphere affecting harmful damage to environment.

We focus the unused energy of discharging cooling water for transforming useful energy with Cavitation phenomenon of water. Cavitation is an inevitable phenomenon that occurs often in hydrodynamic machine or draft tube of hydroelectric generator[1]. In hydro machine, the abrupt event causes severe damage to surface of hydro-blades operating below saturated vapor pressure. In the short moment, internal energy of combined water molecules makes free movement of molecules for creating bubbles and they implode to one point on a surface if the collision cannot support pressure by surrounding liquid[2]. Since the implosion is in fact rapid concentration of molecules to one point, it causes intense shockwave. At the concentration process, if we placed a nano-sized turbine generator in the passing of moving molecules, it could make electricity.
Converging-Diverging nozzle is mainly used in compressible gas jet industry. However, if liquid water passes through the nozzle, some of the liquid boils partially so that we can see mixed two phase flows of water. The void by the nozzle boiling is also a cavitation that is little different from undesirable cavitation. The induced cavitation makes the volume expanded in the fixed nozzle space so that it let average velocity of flow increased. Since fluid is simply moving group of molecules colliding each other, each molecule has its own colliding velocity. Therefore, we have been studyed each molecular velocity to achieve maximum kinetic energy by collision based on conservation of momentum.

Since molecules in the liquid state are binding with neighboring molecules tightly, we cannot approach to the velocity of each molecule. However, it is possible that the water molecules generate cavitation bubbles so that we can approach to each velocity of molecules, when the liquid pass through the nozzle and go into vacuum. Then, we might think of getting momentum by colliding with rotating turbine blades.

In this paper, ideal molecular dynamics simulation for nozzle is introduced for possibilities of getting more energy over high potential energy. Nozzle would be part for separating of liquids and making straight directional flow of molecules. Back pressures in between the nozzle exit area and in front of turbine blades interferes main flow. Since low back pressure means high level of vacuum, moving molecule from nozzle throat may go longer without collisions of random molecules.

Therefore, we simulate nozzle first with molecular dynamics. The goal of this study is investigating whether or not the velocity of molecules at the nozzle exit increases as the vacuum level gets higher. 


\section{Configurations of MD Simulation and Implementation}

Molecular dynamics simulation is performed to study velocity changes according to degrees of vacuum level in the molecular world.

We design a 2-dimensional nano-sized shape of nozzle and implement it. We generate moving molecules with initial velocities in the nozzle. We simulate molecular movements with simple numerical methods such as the Verlet integrations and the Lennard-Jones potentials. Instead of water molecules, argon molecules are used in this simulation. Since argon molecule is symmetric and monatomic, it is desirable for a starting stage. To grasp general tendency of pressure and velocity changes, 2dimensional simulation is used with a personal computer.

\subsection{Nozzle geometry and measurement positioning}

Nozzle geometry for our molecular dynamics simulation is converging-diverging type as shown in Fig. 1. The converging region is marked as $\mathrm{A}$ and the straight moving region after diverging is marked as B. To measure static pressures, we select the arc $\mathrm{L}$ and the line $\mathrm{M}$ for the front part and the rear part of the nozzle throat, respectively. The vertical line $\mathrm{N}$ is selected to measure dynamic pressure and average exit velocity.

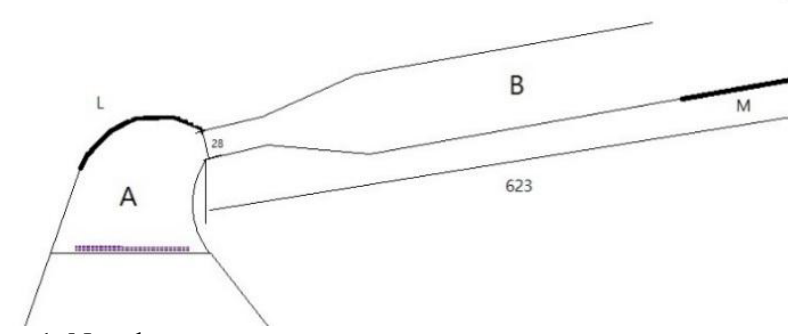

Figure 1. Nozzle geometry

We define base unit as the same size of diameter for one molecule. The length from the throat to exit is 623 base units. The throat diameter is 28 base units and the diverging angle of expansion part is $12^{\circ}$. For a argon molecule, 623 base units is 212 nanometers in physical dimension.

\subsection{Defining pressures for regions $A$ and $B$}

Static pressure is normal force per unit area that we usually use it with pressure gage in fluid machinery. To find out pressure drop when the fluid is passing throat, we define two dimensional pressure which is sum of collision force with chosen line divided by length of the chosen line. Because this simulation is $2 \mathrm{D}$, it is computed as follows:

$$
P_{2 d} \cong \frac{\sum m_{i} a_{i}}{\text { lengt } h \text { of chosen curve or line }}
$$

The $\operatorname{arc} \mathrm{L}$ is a semi-circle before the throat. The length of the $\operatorname{arc~} \mathrm{L}$ is equal to the length of the selected line $\mathrm{M}$.

Dynamic pressure is the kinetic energy of fluid particles per unit volume. To compute the dynamic pressure for the vertical line $\mathrm{N}$, we get the exit velocity of each particle passing through the vertical line $\mathrm{N}$ and calculate the dynamic pressure. Let $q_{2 d}$ be pressure for the vertical line $\mathrm{N}$. It is expressed as follow:.

$$
q_{2 d}=\frac{1}{2} \Sigma \rho v_{i}^{2}
$$

\subsection{Placing molecules for pressure difference configuration}

The most important target of this study is investigating whether or not the velocity of molecules at the nozzle exit increases as the vacuum level gets higher. Since the vacuum level is closely related to the number of molecules in the nozzle, we set the number of molecules generated in region $\mathrm{A}$ to be different from the number of molecules in region $\mathrm{B}$ to have different vacuum levels. That is, we assign different number of molecules to regions $\mathrm{A}$ and $\mathrm{B}$ to keep 4000 molecules difference for experimental sets. Molecules in region A are created with random velocities at the bottom of the nozzle and they collide with each other and the closed walls. Molecules in region $B$ are generated with random velocities at end of the nozzle, to exert some level of pressure on region between the nozzle exit and the rotating blades. These random molecules generated in region B behave as obstructer to the moving fluids to the nozzle exit.

\subsection{Collisions of molecules with sloped nozzle walls}

Here, the colliding nozzle walls in Fig. 1 are sloped. So, the normal force won't be along the conventional Y axis. It makes sense to resolve the velocity along the normal and tangential directions of point of contact. While the velocity along the tangential component would remain the same, the velocity along the normal would reverse in direction. Collisions of molecules with sloped nozzle walls are implemented to calculate new velocities[3].

\subsection{The Lennard-Jones potential and Verlet integration}

The Lennard-Jones Potential is a mathematically simple model that approximates the interaction between a pair of molecules[4]. The Lennard-Jones Potential formula is expressed as follows:

$$
\mathrm{V}_{\mathrm{LJ}}=4 \varepsilon\left[\left(\frac{\sigma}{\mathrm{r}}\right)^{12}-\left(\frac{\sigma}{\mathrm{r}}\right)^{6}\right]
$$

where $\varepsilon$ is the depth of the potential well, $\sigma$ is the finite distance at which the inter-molecule potential is zero, $r$ is 
the distance between the molecules. The attractive $r^{-6}$ term in Eq. (3) represents the long-range behavior of the van der Waals force between molecules. The attractive $r^{-12}$ term represents repulsive particles in this simulation system. force. This is implemented to calculate the potential between molecules. Verlet integration is a numerical method used to integrate Newton's equations of motion[5,6]. It is used to calculate trajectories of molecules

\subsection{Implementing the cell list method}

As algorithm that scales with the number of molecules is the cell list method[7,8,9,10]. The idea of the cell list is illustrated in Fig.2. Region of nozzle is divided into cells with a size slightly larger than the cutoff radius $r_{c}$. Each particle in a given cell interacts with only those particles in the same or neighboring cells. Since the allocation of a particle to a cell is an operation that scales with the number of molecules and the total number of cells that needs to be considered for the calculation of the interaction is independent of the system size, the cell list method scales as with the number of molecules. The cell list method is implemented to save CPU times.

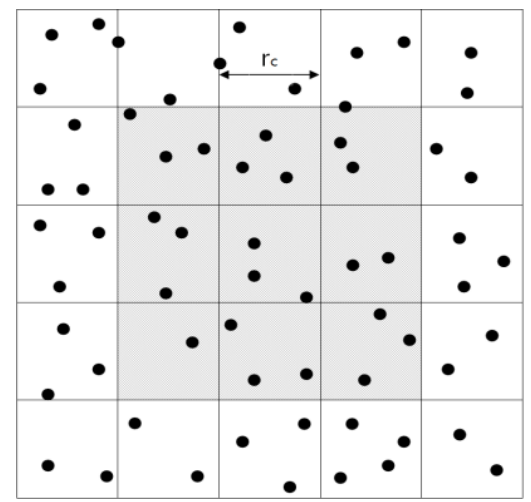

Figure 2. The Cell List (in 2D, 9 neighboring cells)

\section{Result of the simulation}

\subsection{Running the simulation}

The nozzle experiment is shown in Figure3. The number of molecules in regions A and B is 8000 and 4000, respectively and the random velocity generated is around $10 \mathrm{~m} / \mathrm{s} \sim 15 \mathrm{~m} / \mathrm{s}$. As starting the simulation, molecules in region A become equilibrium state after 1 nanosecond and start to pass the nozzle throat. Generated molecules moving in region $\mathrm{B}$ for static pressure at the exit play well as obstacles. After 1.5 nanosecond, we can see the moving flow by moving the group in nozzle but collide soon with the random molecules. Static pressure in the line $\mathrm{M}$ is lculated by the (1). In low pressure area $\mathrm{B}$, we observe that very active clustering occurs and see clear clusters as shown in Fig. 3, after 2 nanosecond. They are moving to the exit area as colliding with neighboring particles. Hardware specification for running the simulation is shown in Table 1.
Table 1. Hardware specification

\begin{tabular}{|l|l|}
\hline & System Information \\
\hline \hline CPU & intel i-7 extream 5960X (haswell-E) \\
\hline RAM & DDR4 PC4-22400 CL15 (8Gx2) x2 = 32GB \\
\hline GPU & $\begin{array}{l}\text { NVIDIA GEFORCE GTX1080 super } \\
\text { JETSTREAM D5X 8GB }\end{array}$ \\
\hline
\end{tabular}

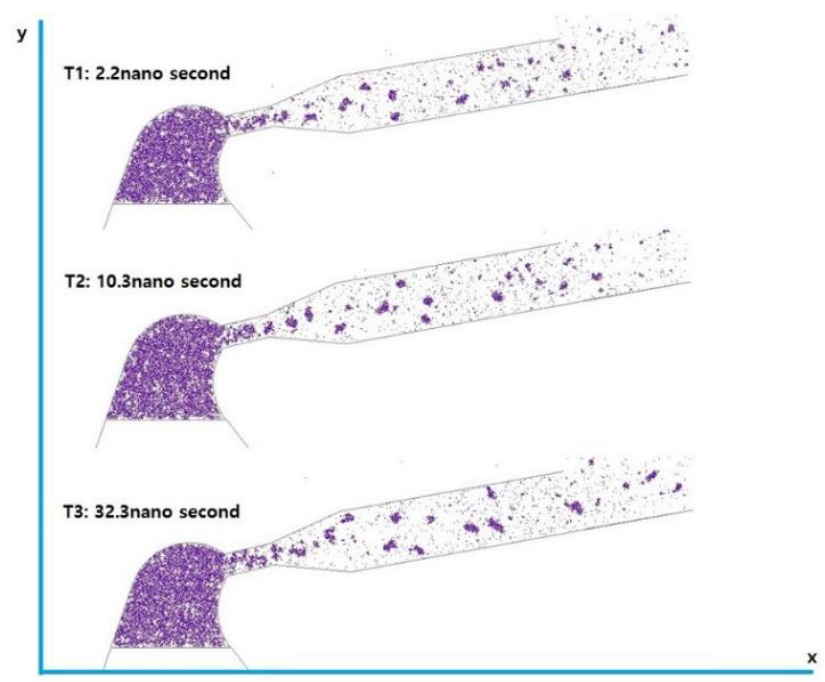

Figure 3. Running the simulation

\subsection{Case study according to vacuum levels}

The most important target of this simulation is analyzing static and dynamic pressure changes and their general trend in case study of different pressure configuration in regions $\mathrm{A}$ and $\mathrm{B}$. The experimental sets are shown in Table 2 for the configuration. To see static pressures of these regions, we directly compare $P_{2 d}$ in Figure4. The graph shows that the pressure in the arc L is higher than the pressure in the line $\mathrm{M}$. the pressure difference of the arc $\mathrm{L}$ and the line $\mathrm{M}$ is more than 10 time in the nozzle.

Figure 5 shows static pressure comparison at L part, the semi-circle before throat of nozzle. The graphs show general trend of pressure in converging part of the nozzle. The more number of molecules, the more number of collisions are detected in the simulation.

Figure 6 shows that static pressure comparison at M part, the line part after throat of nozzle. The graphs show general trend of pressure in diverging part of the nozzle. The more number of molecules, the more number of collisions are detected in the simulation. Different from Figure 5, however, trend of graph according to the number of random molecules for back pressure setting shows interesting results.

Figure 7 shows that dynamic pressure comparison at $\mathrm{N}$ part, the vertical line part near the exit of the nozzle. The graphs show general trend of dynamic pressures that can convert useful power. The more number of molecules, the more number of collisions are detected in the beginning stage. However, according to the number of random molecules for back pressure setting, very different results are acquired. When random molecules of back pressure collide with directional molecules, the dynamic pressure 
reduced. Even though this simulation setting can be ideal, it is evident that the vacuum level affect to the velocity of exit. The numerical setting is that for each case, they have the same number of molecules that is they have same difference of pressure

Table 2 Number of molecules for regions A and B

\begin{tabular}{|l|l|l|l|l|l|}
\hline Case & 8000 by 4000 & 7000 by 3000 & 6000 by 2000 & 5000 by 1000 & 4000 by 0 \\
\hline Symbol & - & $\cdots \cdots$ & -- & $-\cdot$ & $-\cdot \cdot$ \\
\hline
\end{tabular}

Captions should be typed in 9-point Times. They should be centred above the tables and flush left beneath the figures.
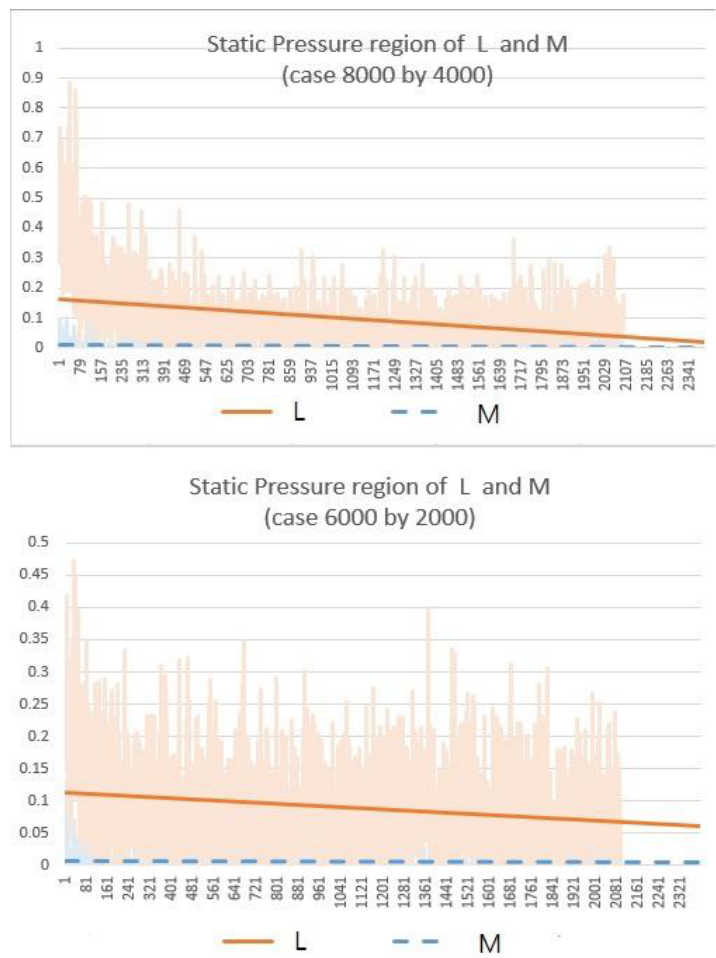

Figure 4. Static Pressures of regions L and M

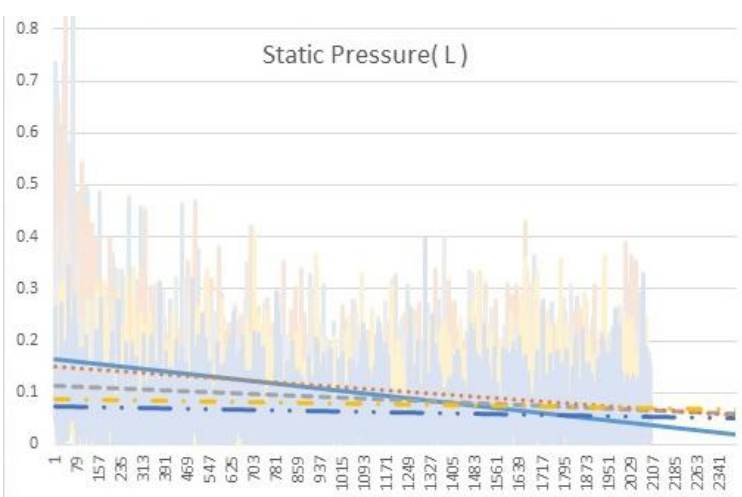

Figure 5. Static Pressure Comparison at $\mathrm{L}$ region

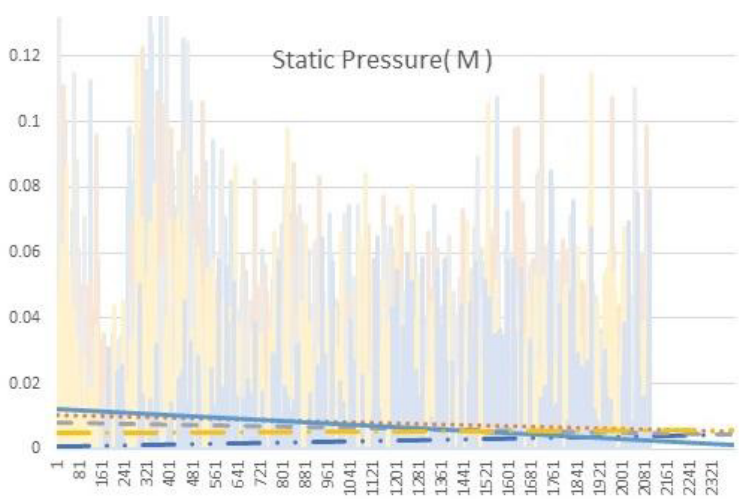

Figure 6. Static Pressure Comparison at M region

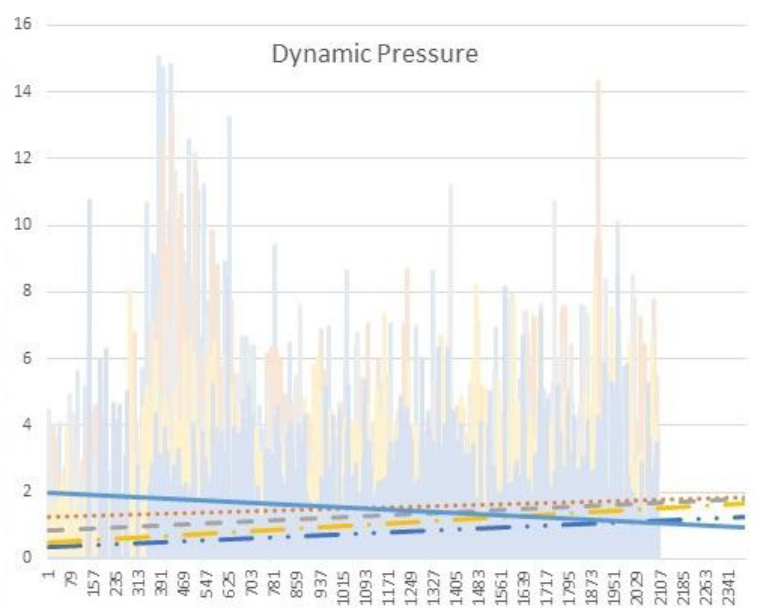

Figure 7. Dynamic Pressure Comparison near N region

\section{Review of the clustering and increasing mean velocity}

\subsection{Clustering in the nozzle}

When high-pressured liquid expands to vacuum region, it is difficult to think for fluid dynamics researchers that the molecules are gathered each other and become state like liquid. However, in the experiments in high-degree of vacuum space, we can observe a new phenomenon of clustering. The clustering is made by the Lennard-Jones potential, which is weak attraction between near molecules. If the molecules are expanded to vacuum but governed by specially designed shape of wall, they close to each other in same directional flow, so that they gather and are weakly bonded. Fig.3 shows well developed clusters. Nuclear physics researchers use the clustering phenomenon to major relative velocity of ion-beam[11]. In view of conservation of momentum, it is expected that if the clustered molecules collide with fixed object, then they bound with scattered gas. However, if they collide to moving object with relatively long time, they might lose the momentum and reduce their velocities. Fig.3 and Fig.7 show the degree of clustering according to vacuum level. Degree of clustering related to moving mass increase without loss of velocity by collision in equilibrium space. 


\subsection{Increase of average velocity of the flow}

The average velocity increase appeared in Fig.8, accordin $\mathrm{g}$ to vacuum level. In real physics, pressure in near exit of nozzle means disturbing the flow by the random moving. Therefore the collision less moving group gives possibilit $\mathrm{y}$ of converting power by the rotating turbine and electric generator.

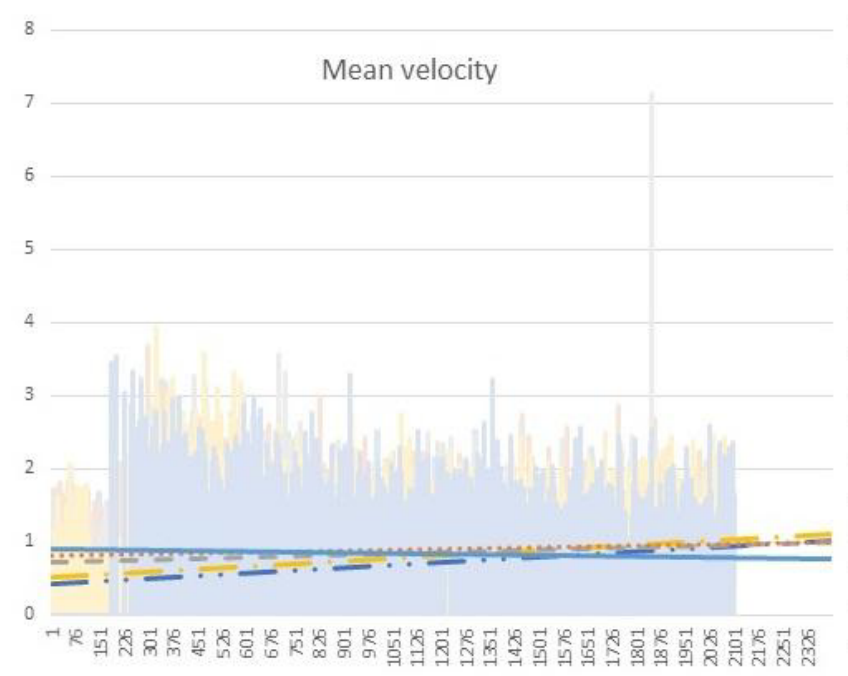

Figure 8. Mean Velocity Comparison near $\mathrm{N}$ region

\section{Conclusion}

We simulated the pressures and velocities of moving molecule in a nozzle to approach each molecule's velocity. Different from general molecular study of highpressure jet, we performed case study of nozzle eruption according to degree of vacuum level. The result shows that the velocities are increased at the nozzle exit as the vacuum level gets higher in that area because less molecular collisions.

\section{Future works}

To use this study in engineering scale, enlarging of nozzle size at least a micro level, and modifying the shape of nozzle for better performance are essential. So, we started developing for the code in graphic user interface environment. In diverging part of the nozzle, we are going to investigate relationship between diverging shape and speed of the flow to out-normal direction. According to the increasing nozzle size, a huge number of molecular collisions are inevitable problem so that we plan to acclerate the calculation code with GPU before using supercomputer.

\section{References}

1. S. Khurana, Navtej and Hardeep Singh, "Effect of Cavitation on Hydraulic Turbines-A Review," INPRESSCO(2012).

2. M. Parlange, M. Farhat, O.Minster, D.Obreschkow, J.R. Thome, "The effect of pressure gradient on the collapse of cavitation bubbles in normal and reduced gravity," 22-23, 2013

3. D. John Cutnell and W. Kenneth Johnson, "Essential of Physics," John Wiley \& Sons, Inc., 2006.

4. Lennard-Jones, "On the Determination of Molecular Fields", Proc. R. Soc. Lond. A, 106 (738): 463-477, 1924

5. Verlet, Loup, "Computer "Experiments" on Classical Fluids. I. Thermodynamical Properties of Lennard-Jones Molecules". Physical Review. 159: 98-103, 1967.

6. V. Daniel Schroeder, "Interactive molecular dynamics,"Am. J. Phys., Vol. 83, No. 3, 210-218, March 2015.

7. D. Frenkel and B. Smit, Understanding Molecular Simulation(Academic, New York, 2002).

8. B. R. Holstein, "The van der Waals interaction," Am. J. Phys. 69(4), 441-449 (2001).

9. K. A. Milton, "Resource Letter VWCPF-1: van der Waals and Casimir-Polder forces," Am. J. Phys. 79(7), 697-711 (2011).

10. G. C. Maitland et al., Intermolecular Forces: Their Origin and Determination (Clarendon Press, Oxford, 1981). This monograph makes an exhaustive assessment of the limitations of the Lennard-Jones 612 potential.

11. A. Giannuzzi; F.A. Stevens (2004). Introduction to Focused Ion Beams: Instrumentation, Theory, Techniques and Practice. Springer Press 